\title{
Article \\ Error Analysis of Integrated Absorbance for TDLAS in a Nonuniform Flow Field
}

\author{
Renjie $\mathrm{Li}^{1,2}{ }^{1}$, Fei $\mathrm{Li}^{1}{ }^{1}$, Xin Lin ${ }^{1, *}$ and Xilong $\mathrm{Yu}^{1,2}$ \\ 1 State Key Laboratory of High Temperature Gas Dynamics, Institute of Mechanics, Chinese Academy of \\ Sciences, Beijing 100190, China; lirenjie@imech.ac.cn (R.L.); lifei@imech.ac.cn (F.L.); xlyu@imech.ac.cn (X.Y.) \\ 2 School of Engineering Science, University of Chinese Academy of Sciences, Beijing 100049, China \\ * Correspondence: linxin_bit@imech.ac.cn
}

Citation: Li, R.; Li, F.; Lin, X.; Yu, X. Error Analysis of Integrated Absorbance for TDLAS in a Nonuniform Flow Field. Appl. Sci. 2021, 11, 10936. https://doi.org/ 10.3390/app112210936

Academic Editor: Wolfgang Elsaesser

Received: 9 October 2021

Accepted: 16 November 2021

Published: 19 November 2021

Publisher's Note: MDPI stays neutral with regard to jurisdictional claims in published maps and institutional affiliations.

Copyright: (c) 2021 by the authors. Licensee MDPI, Basel, Switzerland. This article is an open access article distributed under the terms and conditions of the Creative Commons Attribution (CC BY) license (https:// creativecommons.org/licenses/by/ $4.0 /)$.

\begin{abstract}
As an effective optical diagnosis method, tunable diode-laser absorption spectroscopy (TDLAS) has increasingly moved to examine nonuniform flows, such as two-dimensional combustion diagnosis. To investigate the effect of nonuniformity along the line of sight in a measurement using TDLAS, the integrated absorbance (IA, the key intermediate quantity in TDLAS) error was quantified. The error distribution is obtained from the line-shape parameters through the comprehensive analysis of the line-shape function and the fitting method. The effects of the fitting function and the absorption line overlap are also considered. A general method for estimating the error is given. The work illustrates the applicability of TDLAS technology in nonuniform flow fields and provides input parameters for the evaluation of tunable diode laser absorption tomography error.
\end{abstract}

Keywords: TDLAS; integrated absorbance; error analysis

\section{Introduction}

Tunable diode laser absorption spectroscopy (TDLAS) technology has a long history of use in the high-temporal-resolution, noninvasive, and quantitative measurements of the gas temperature, pressure, and composition in many practical, near-homogenous environments (e.g., environments of the atmosphere, shock tubes, scramjets, and internal-combustion engines) $[1,2]$. However, because many flows of practical interest have strong nonuniformity, several researchers have advanced strategies for nonuniform flows to obtain the flow field parameters. Past efforts can be broadly divided into two categories [3-5]. Efforts in the first category improve the TDLAS to obtain the parameter distribution information along the optical path [6-8]. Efforts in the second category obtain the spatial resolution by combining TDLAS with tomography. This combination has resulted in the tunable diode laser absorption tomography (TDLAT) method. This method is mainly applied to combustion and reaction flow and is a two-dimensional measurement technique with great potential [9-16].

Most TDLAS technology used in a nonuniform flow field, especially TDLAT technology, adopts direct absorption spectroscopy [14]. Figure 1 illustrates the application of direct absorption TDLAS in a nonuniform flow field. A spectral absorption signal is obtained after the laser light is absorbed. The absorption signal undergoes data processing (line-shape fitting) to obtain the integrated absorbance (IA; i.e., $A$ ). Depending on a few key parameters (including pressure, line parameters, etc.), measurement results are obtained through the tomographic calculation of the IA. The IA is the key intermediate quantity in the measurement process. However, in a nonuniform flow field, the nonuniformity of the flow field is coupled with the absorption line features (including the spectroscopic parameters and absorption line overlap) and the measurement noise, resulting in distortion of the measured absorption signal. The distorted absorption signal interferes with the line-shape fitting, resulting in an IA error, which ultimately affects the measurement result. It is therefore necessary to study the IA error generated by signal distortion in a nonuniform flow field. 


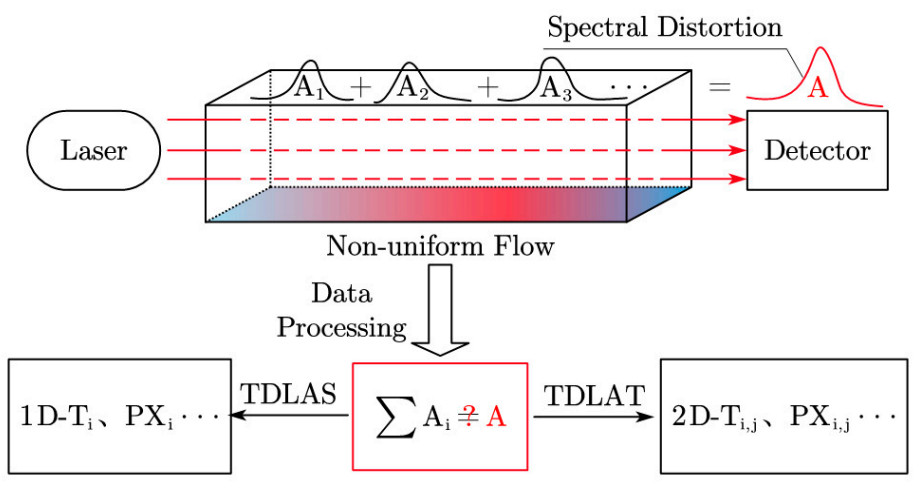

Figure 1. Schematic of TDLAS measurement in a nonuniform flow field.

Previous studies identified the nonuniformity of the flow field (especially the boundary layer effect) as an important factor affecting the accuracy of TDLAS measurement [17-20]. Xiang et al. proposed an energy-temperature curve for estimating the effects of thermal and concentration boundary layers and assisting the selection of candidate absorption lines [17]. Li et al. quantified the uncertainty generated by various nonuniformities typically encountered in a velocity measurement [18]. Qu et al. quantified the effects of the temperature gradient and thermal boundary layer thickness on the spectral collisional width and gas concentration measurement [19]. Ma et al. quantified the uncertainty in the measurement of the TDLAS-determined temperature [20].

The above research provides a basis for the applicability of TDLAS in one-dimensional measurement of nonuniform flow field. However, when including multiple factors simultaneous (including IA, line parameters, pressure, etc.) in the study, there are challenges to apply TDLAS technology in nonuniform flow fields as a universal method. It is also difficult to perform general analysis of TDLAS measurement errors, especially to evaluate the uncertainty of two-dimensional measurement, which requires separate error analysis of IA. However, only a few researchers have studied the IA error. S. T. Sanders et al. proposed a hybrid line-shape fitting method, which combines direct integration and line-shape fitting to obtain the IA more accurately [6]. Liu et al. applied this method in a nonuniform flow field measurement [7]. Nie et al. used a series of low-temperature water absorption lines to further analyze the use of this method [21]. C. S. Goldenstein considered that the spectral signal can be freely fitted using empirical line-shape parameters with the Voigt model and that the best fitting result can be selected [8]. These methods provide a more accurate IA but do not quantify the IA systematic error generated by nonuniformity.

Other researchers have reported the measurement uncertainty generated by line-shape fitting [22-24]. Such work can be regarded as a quantification of IA uncertainty but not a quantification of the systematic error generated by the nonuniformity. The latter should be analyzed and compensated for in the measurement. There has been no quantitative analysis of the IA error for a nonuniform flow field. Due to the complexity of the nonuniform flow field and the diversity of available absorption lines, it is difficult to comprehensively and accurately evaluate the system error of IA and judge the applicability of TDLAS technology to nonuniform flow.

This paper studies the IA error under a nonuniform flow field. Measurement noise varies with changes in the test system, so this variation is not considered in the current study. We focus here on the IA error generated by the coupling of the nonuniformity, the absorption line features, and the line-shape fitting function, which are inherent to the measurement. The paper analyzes the effect of nonuniformity on the IA error from the perspective of line-shape parameters, removing the limitation of a specific nonuniform flow field and specific absorption line. The IA error generated by the nonuniformity and the isolated absorption line are universally quantified. Finally, considering the effect of the absorption line overlap in combination with practical applications, a general method for estimating the IA error is given. 


\section{Materials and Methods}

\subsection{Line Shape Theory}

A detailed description of TDLAS technology can be found in the literature $[25,26]$. A brief summary is provided here to facilitate the discussion in subsequent sections. Along a uniform-property path, the narrowband absorption of a light signal at wavelength $v\left(\mathrm{~cm}^{-1}\right)$ is described by the Lambert-Beer Law:

$$
\begin{gathered}
a=\ln \frac{I_{0}(v)}{I_{t}(v)}=A \Phi(v), \\
A=\int_{-\infty}^{+\infty} \ln \frac{I_{0}(v)}{I_{t}(v)} d v=P X_{m} S_{m}(T) L \int_{-\infty}^{+\infty} \Phi(v) d v,
\end{gathered}
$$

where $a$ is the spectral absorbance, $A$ is the IA (i.e., the area under the curve), $I_{0}(v)$ and $I_{t}(v)$ are the incident light intensity and the transmitted light intensity, respectively, $P X_{m}$ is the partial pressure of absorber $m, S_{m}(T)$ is the line strength, $L$ is the absorption length, and $\Phi(v)$ is a line-shape function whose integral is normalized to unity $\left(\int_{-\infty}^{+\infty} \Phi(v) d v=1\right)$.

In the case of a normal analytical source, the line shape depends on contributions from collisional (Lorentzian) and Doppler (Gaussian) broadening [27]. The spectral line-shape is a convolution of Lorentzian and Gaussian line shapes and can be described by a Voigt profile [28] given by

$$
\begin{gathered}
\Phi_{V}\left(v, v_{0}\right)=\frac{1}{\sqrt{\pi}} x^{\prime} K(x, y), \\
K(x, y)=\frac{y}{\pi} \int_{-\infty}^{+\infty} \frac{1}{(x+t)^{2}+y^{2}} e^{-t^{2}} d t,
\end{gathered}
$$

where $x$ is the normalized wave number, $x^{\prime}$ is the normalized coefficient, and $y$ is the line damping parameter, which controls the line shape. The three line-shape parameters $\left(x, x^{\prime}\right.$ and $y$ ) are given by

$$
\begin{gathered}
x=x^{\prime} v^{\prime}, \\
v^{\prime}=v-v_{0}-v_{c} \\
x^{\prime}=\frac{\sqrt{\ln 2}}{\alpha_{D}}, \\
y=\sqrt{\ln 2} \frac{\alpha_{L}}{\alpha_{D}},
\end{gathered}
$$

where $v^{\prime}$ is the relative wavenumber, $v_{0}$ is the wavenumber at the line center, $v_{c}$ is the pressure/velocity-induced frequency shift of the absorption line profile with respect to the unperturbed wavenumber $v_{0}$, and $\alpha_{D}$ and $\alpha_{L}$ are respectively the Doppler and collisional half-widths at half-maximum (HWHMs). The two HWHMs are affected by environmental and spectroscopic parameters:

$$
\begin{gathered}
\alpha_{D}=v_{0} \sqrt{\frac{2 k_{B} T \ln 2}{M c^{2}}}, \\
\alpha_{L}=\sum_{g} P X_{g} \gamma_{g}\left(\frac{T_{0}}{T}\right)^{n_{g}} .
\end{gathered}
$$

Here, $k_{B}$ is the Boltzmann constant, $M$ is the molecular weight of the absorbing species, $c$ is the speed of light, $T$ is the gas temperature, $P X_{g}$ is the partial pressure of the collision partner of the absorbing-species, and $\gamma_{g}$ and $n_{g}$ are the collisional-broadening coefficient and temperature-dependent exponent of the species $g$, respectively. $\gamma_{g}$ and $n_{g}$ are both spectroscopic parameters that can be queried in a database (e.g., HITRAN 2016 [29]). 


\subsection{Error Analysis}

\subsubsection{Simulation of the Nonuniform Absorption Signal}

In most practical applications, the overlap of absorption lines interferes with the measurement. Generally, isolated absorption lines should be selected as far as possible [30]. For the isolated absorption line, it is seen from the line-shape function [Equations (3)-(10)] that the absorption signal is affected by eight parameters (including flow field and spectroscopic parameters). However, the absorption signal can be characterized by five line-shape parameters, namely the $A$, normalization coefficient $x^{\prime}$, line damping parameter $y$, absorption line center wavenumber $v_{0}$, and frequency shift $v_{c}$.

In the case of a nonuniform flow field, the absorbance is the integral of the absorption length $L$. Mathematically, the line-shape function is nonlinear, and therefore, the nonuniform absorption signal cannot be characterized by a single uniform absorption signal, i.e., the nonuniformity of the flow field leads to the distortion of the line shape. Equation (1) is rewritten as

$$
a=\ln \frac{I_{0}(v)}{I_{t}(v)}=\int_{0}^{L} P X_{m}(l) S_{m}(T(l)) \Phi(v, l) d l .
$$

The integral of Equation (11) can be regarded as the linear superposition of $n$ quasiuniform flow field absorption signals. When an isolated absorption line is determined to be used and the pressure and velocity of the flow field do not change greatly, changes in the center wavenumber $v_{0}$ and frequency shift $v_{c}$ may be disregarded. The remaining three line-shape parameters are used to write the absorption signal as

$$
a=\sum_{i=1}^{i=n} A_{i} \frac{1}{\sqrt{\pi}} x_{i}^{\prime} K\left(x_{i}^{\prime} v^{\prime}, y_{i}\right)
$$

Equation (12) is the functional expression of the absorption signal of a general nonuniform flow field. This means that for the measurement using any isolated spectral absorption line in any flow field without a frequency shift, the spectral absorption signal can be modeled using the line-shape parameters (i.e., the line damping parameter $y_{i}$, normalization coefficient $x^{\prime}{ }_{i}$, and $A_{i}$ ) of $n$ quasi-uniform flow field regions.

Therefore, the theoretical truth of the IA is

$$
A_{S}=\sum_{i=1}^{i=n} A_{i}
$$

\subsubsection{Fitting of the Absorption Signal}

In data processing, it is necessary to perform line-shape fitting on the absorption signal to obtain the IA. The fitting of the absorption signal can be transformed into the solving of a nonlinear least-squares problem. The essence of the problem is to obtain a set of parameters $\beta$ such that $\chi^{2}(\beta)$ is a minimum value:

$$
\chi^{2}(\beta)=\sum_{j=1}^{j=n}\left[a_{j}-F\left(v_{j}, \beta\right)\right]^{2},
$$

where $a_{j}$ is the absorbance corresponding to the absorption signal data point $j$ and $v_{j}$ is the corresponding wavenumber, which is simulated using Equation (12) in this work. $F(v, \beta)$ is the regression function, which is a single Voigt line-shape function in this work.

In most practical applications, because $\alpha_{D}$ (i.e., the Doppler HWHM) and $\alpha_{L}$ (i.e., the collisional HWHM) have clear physical meanings, they are generally used as parameters for fitting [31]. To facilitate the analysis, this work uses the normalized coefficient $x^{\prime}$ and the 
line damping parameter $y$ instead of the two HWHMs as parameters for fitting. Therefore, the fitting parameters $\beta=\left(v_{f}, A_{f}, x_{f}^{\prime}, y_{f}\right)$ and the regression function are written as

$$
F\left(v, \beta=\left(v_{f}, A_{f}, x_{f}^{\prime}, y_{f}\right)\right)=A_{f} \frac{1}{\sqrt{\pi}} x_{f}^{\prime} K\left(x_{f}^{\prime}\left(v-v_{f}\right), y_{f}\right) .
$$

To solve the above problem, this work uses the Levenberg-Marquardt fitting algorithm (L-M algorithm), which is widely used in most absorption analyses [32]. A more detailed introduction to the fitting algorithm can be found in the literature [33]. In actual calculations, the L-M algorithm has a certain dependence on the initial values of the fitting parameters. To achieve the best fitting effect, this work combines the calculation methods of Doppler and the collisional HWHM in reference [34] to pre-calculate the initial values of fitting parameters.

The Voigt function has no analytical solution, and there are thus a variety of numerical methods and approximation formulas with which to calculate it [27,35-37]. The calculation speed and accuracy of the approximation methods affect the solution of IA. Considering the calculation accuracy and speed, this work uses the 916 algorithm [38] to perform the high-precision simulation of the Voigt line shape. A Voigt line-shape function approximate calculation method (Fadf algorithm) proposed by S. M. Abrarov is then used to fit the absorption signal [39-42]. The Fadf algorithm is based on a Fourier expansion and Laplacian continuous fractions that enable a fast approximation of the Voigt function.

The fitting parameter $A_{f}$ obtained after the fitting iteration is the measured value of the IA. Combined with the theoretical true value $A_{S}$ of the IA, the relative error of the IA (i.e., the IA error) is obtained as

$$
R=\frac{A_{f}-A_{S}}{A_{S}} .
$$

\subsubsection{Error Analysis under a Nonuniform Flow Field}

In a nonuniform flow field, the IA error is affected by multiple physical quantities, which is the difficulty of error analysis. Based on the analysis in the previous section, we can find that it may be simple to analyze the error from the perspective of line-shape parameters. At this time, we only need to consider three parameters (i.e., the line damping parameter $y_{i}$, normalization coefficient $x_{i}^{\prime}$, and $A_{i}$ ). Focusing on the absorption signal of the general nonuniform flow field, the effects of the line-shape parameters on the IA error are analyzed with the line-shape fitting method. For the convenience of analysis, the number of quasi-uniform flow field areas is set at two. The corresponding physical background is a flow field with a boundary layer. By substituting Equations (12) and (13) into Equations (14) and (16) yields, we have,

$$
\begin{gathered}
\chi^{2}\left(\beta=\left(v_{f}, A_{f}, x^{\prime}, y_{f}\right)\right)=\sum_{j=1}^{j=n}\left[\begin{array}{c}
A_{f} \frac{1}{\sqrt{\pi}} x^{\prime}{ }_{f} K\left(x^{\prime}{ }_{f} v^{\prime}, y_{f}\right)- \\
\left(A_{1} \frac{1}{\sqrt{\pi}} x^{\prime}{ }_{1} K\left(x^{\prime}{ }_{1} v^{\prime}, y_{1}\right)+A_{2} \frac{1}{\sqrt{\pi}} x^{\prime}{ }_{2} K\left(x^{\prime}{ }_{2} v^{\prime}, y_{2}\right)\right)
\end{array}\right]^{2} . \\
A_{f} \frac{1}{\sqrt{\pi}} x^{\prime}{ }_{f} K\left(x^{\prime}{ }_{f} v^{\prime}, y_{f}\right)- \\
R=\frac{\int_{-\infty}^{+\infty}\left(A_{1} \frac{1}{\sqrt{\pi}} x^{\prime}{ }_{1} K\left(x^{\prime}{ }_{1} v^{\prime}, y_{1}\right)+A_{2} \frac{1}{\sqrt{\pi}} x^{\prime}{ }_{2} K\left(x^{\prime}{ }_{2} v^{\prime}, y_{2}\right)\right) d v_{j}}{\int_{-\infty}^{+\infty} A_{1} \frac{1}{\sqrt{\pi}} x^{\prime}{ }_{1} K\left(x^{\prime}{ }_{1} v^{\prime}, y_{1}\right)+A_{2} \frac{1}{\sqrt{\pi}} x^{\prime}{ }_{2} K\left(x^{\prime}{ }_{2} v^{\prime}, y_{2}\right) d v_{j}} .
\end{gathered}
$$

An analysis of Equations (17) and (18) reveals that when $x^{\prime}{ }_{i}$ and $y_{i}$ are unchanged and $A_{i}$ changes by a multiple, the minimum $\chi^{2}(\beta)$ is obtained by multiplying the fitting parameter $A_{f}$ by the same multiple. Therefore, the error $R$ does not change. Similarly, because $x_{i}^{\prime}$ is a line-shape normalization coefficient, when $A_{i}$ and $y_{i}$ are unchanged and $x_{i}^{\prime}$ changes by a multiple, $x_{f}^{\prime}$ obtained by the fitting changes by the same multiple and $A_{f}$ obtained in the fitting does not change. Therefore, the error $R$ does not change. It is easily seen that the above analysis is also applicable for $n>2$. 
Therefore, when the number $n$ of quasi-uniform flow field areas is determined, the error only depends on $y_{i}$ of each quasi-uniform flow field area, the relative ratio $x_{1}^{\prime}: x_{2}^{\prime}$ : $x_{3}^{\prime}: \ldots: x_{i}^{\prime}$ of each $x^{\prime}{ }_{i}$, and the relative ratio $A_{1}: A_{2}: A_{3}: \ldots: A_{i}$ of each $A_{i}$. For the flow field comprising $n$ quasi-uniform flow field areas, the IA error distribution can be calculated from the line damping parameter range, normalized coefficient ratio range, and IA ratio range of each flow field area.

\subsubsection{Typical Range of Line-Shape Parameters}

Calculating the IA error distribution requires the typical ranges of line-shape parameters to be obtained. Section 2 revealed that the ratio of the normalized coefficient is only affected by the temperature, whereas the line damping parameter and the IA are affected by the flow field and spectroscopic parameters. It is thus necessary to obtain the typical ranges of line-shape parameters based on actual measurement conditions.

Taking $n=2$ as an example, for a typical absorption line (i.e., the near-infrared absorption line of water $v_{0}=7185.597 \mathrm{~cm}^{-1}$ ) applied under general measurement conditions (i.e., water as the absorption component, static pressure of $1 \mathrm{~atm}, \mathrm{~T}=200 \mathrm{~K} \sim 4000 \mathrm{~K}$, and $\left.P X_{\mathrm{H}_{2} \mathrm{O}}=0 \mathrm{~atm} \sim 0.4 \mathrm{~atm}\right)$, the ranges of the ratio of the normalization coefficient and the line damping parameter can be calculated. The distributions are shown in Figure 2. The spectral parameters are taken from the HITRAN database. The IA is affected by many factors, and therefore, only the ratio range needs to be considered.
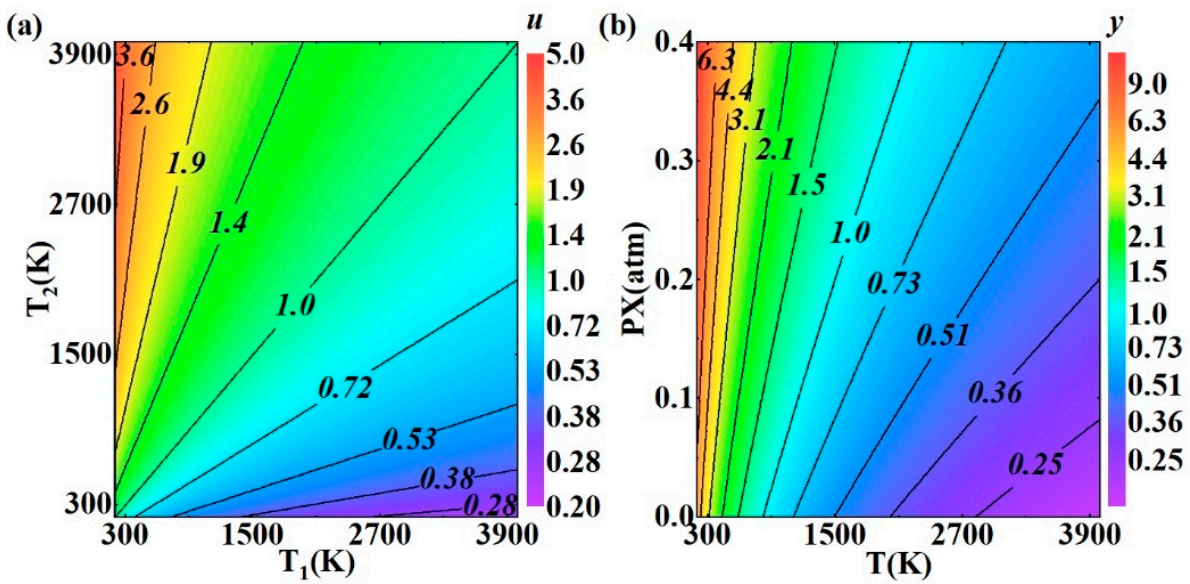

Figure 2. Typical applications: (a) range of the normalization coefficient ratio and (b) range of the line damping parameter.

The absorption line does not affect the normalization coefficient ratio but does affect the line damping parameter range. The calculation results show that by comprehensively considering the changes in spectroscopic parameters and practical applications (e.g., the choice of database under different conditions.), $u=x_{1}^{\prime}{ }_{1} / x_{2}^{\prime}=0.25 \sim 4, y=0.1 \sim 10$, and $A_{1} / A_{2}=0.1 \sim 0.9$ can be taken as the typical ranges of line-shape parameters.

\section{Results and Analysis}

The analysis presented in Section 2 revealed that through the fitting of the absorption signal, an IA error distribution table can be obtained when there are two quasi-uniform flow field regions. (See Supplementary Materials for the IA error distribution table.) The distribution table is the quantified result of the IA error. We consider an analysis for several typical conditions. The IA error has a positive or negative value. For the convenience of analysis, the absolute value of IA error is used in the analysis. This section discusses only the error distribution under nonuniform conditions, and the uncertainty in the spectral parameters is thus not considered. 


\subsection{Case 1: $A_{1} / A_{2}=1$}

Figure 3 shows the distribution of the absolute value of the IA error with a change in the line damping parameter in two quasi-uniform flow field regions when the IA ratio is fixed at 1 and the normalized coefficient ratio is set at 1:1, 1.5:1, 2:1, and 2.5:1. Considering the physical meanings of the parameters, the different cloud images in Figure 3 represent different temperature ratios of the two quasi-uniform flow field regions. Any small cloud image shows a change in the IA error as the nonuniformity of the static pressure or the absorber concentration in the flow field changes when the temperature ratio of the two quasi-uniform flow field regions is fixed and the IA rate is the same. A comparison of the four cloud images in Figure 3 reveals that a larger temperature ratio of the two quasiuniform flow fields results in a smaller area being surrounded by the contour $R=0.01$. In extreme cases, the error generated by the coupling of the nonuniformity and spectroscopic parameters may exceed 0.1 .
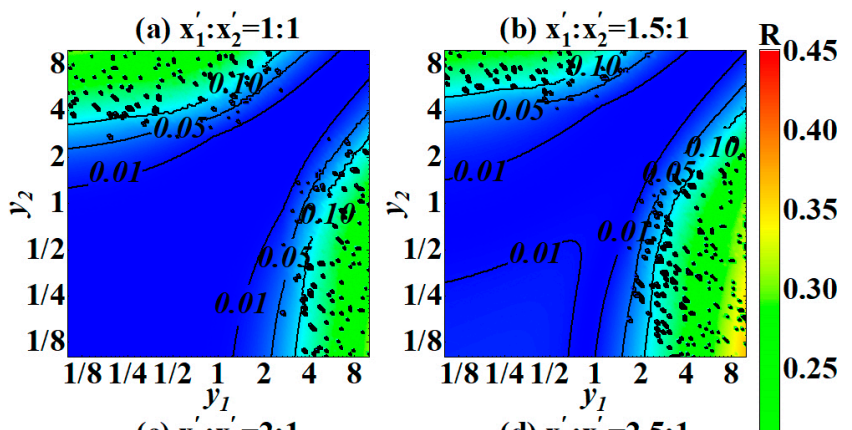

(c) $\mathrm{x}_{1}^{\prime}: \mathrm{x}_{2}^{\prime}=2: 1$
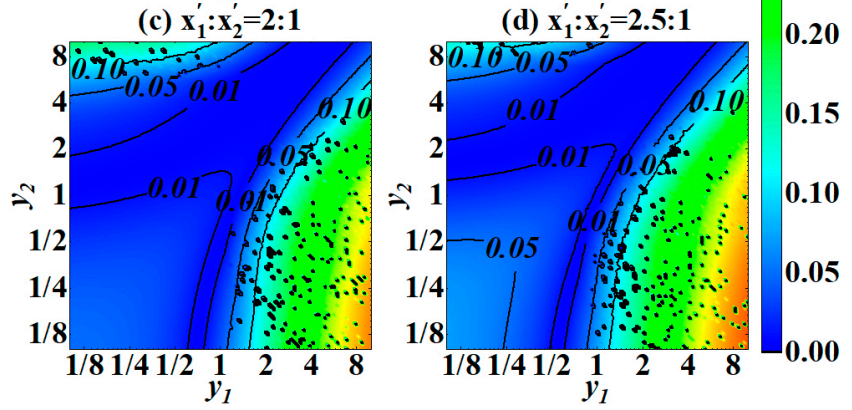

Figure 3. Distribution of the absolute value of the IA error for $A_{1} / A_{2}=1$ and different values of $u=x_{1}^{\prime}{ }_{1} / x_{2}^{\prime}$ : (a) $x_{1}^{\prime} / x_{2}^{\prime}=1: 1$, (b) $x_{1}^{\prime}{ }_{1} / x_{2}^{\prime}=1.5: 1$, (c) $x_{1}^{\prime}{ }_{1} / x_{2}^{\prime}=2: 1$, (d) $x_{1}^{\prime}{ }_{1} / x_{2}^{\prime}=2.5: 1$.

3.2. Case 2: $u=x_{1}^{\prime} / x_{2}^{\prime}=1$

Figure 4 shows the distribution of the absolute value of the IA error with a change in the line damping parameter in the two quasi-uniform flow field regions when the ratio of the normalization coefficient is fixed at 1 and the IA ratio $A_{1} / A_{2}$ is set at 1:0.2, 1:0.4, 1:0.6, and 1:0.8. Additionally, considering the physical meanings of the parameters, the temperature distribution is uniform, and there are only two quasi-uniform flow field regions with different static pressures or absorber concentrations. Different cloud images indicate the relative magnitude of the IA in the two quasi-uniform flow field regions. A comparison of the four cloud images in Figure 4 reveals that the area enclosed by the contour $R=0.01$ decreases as the IA ratio of the two quasi-uniform flow field regions approaches 1 . However, when the IA in one of the quasi-uniform flow field regions occupies most of the field, the nonuniformity of the flow field may still generate large errors $(R>0.01)$. 
(a) $\mathrm{A}_{1}: \mathrm{A}_{2}=1: 0.2$

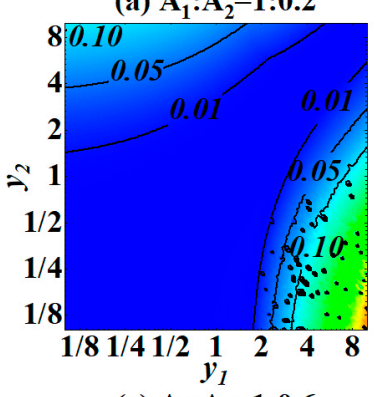

(c) $\mathrm{A}_{1}: \mathrm{A}_{2}=1: 0.6$

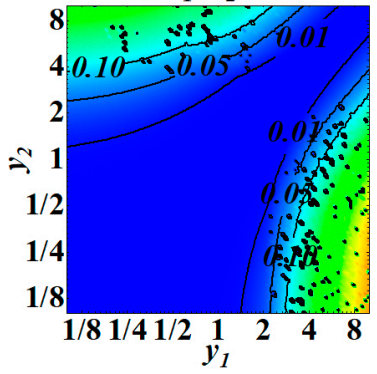

(b) $\mathrm{A}_{1}: \mathrm{A}_{2}=1: 0.4$

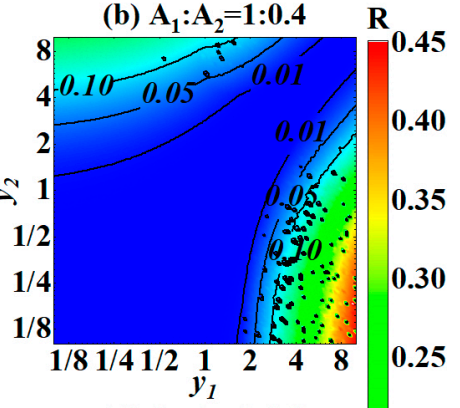

(d) $\mathrm{A}_{1}: \mathrm{A}_{2}=1: 0.8$

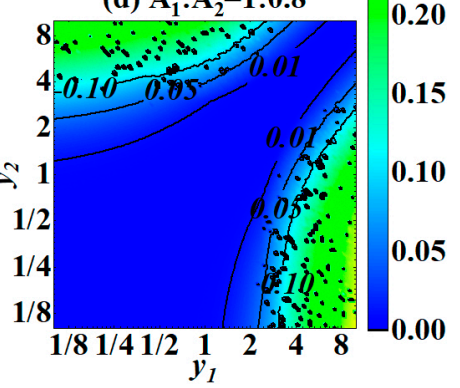

Figure 4. Distribution of the absolute value of the IA error for $u=x^{\prime}{ }_{1} / x^{\prime}{ }_{2}=1$ and different values of $A_{1} / A_{2}:$ (a) $A_{1} / A_{2}=1: 0.2$, (b) $A_{1} / A_{2}=1: 0.4$, (c) $A_{1} / A_{2}=1: 0.6$, (d) $A_{1} / A_{2}=1: 0.8$.

The error in most areas from the bottom left to the top right of the two graphs is less than 0.01. It is seen that the IA error is less than 0.01 when the changes in partial pressure and temperature are not drastic. However, the errors rise sharply as the gap in the line damping parameter between the two quasi-uniform flow field regions increases. It is considered that the changes in the static pressure and the component concentration have a stronger effect on the IA error.

Both images have large amounts of black noise in the area $R>0.01$. A point of black noise is surrounded by a contour line indicating that the error at this point is less than 0.01 , 0.05 , or 0.1 ; i.e., there are points with small errors in areas with large errors. Mathematically, the error distribution should be a smooth surface, and the noise may result from using the Voigt line shape approximated using a numerical algorithm for fitting. Refer to Section 3.4 for a detailed analysis of the effect of the fitting function.

\subsection{Case 3: $y_{1}=y_{2}$}

In some practical applications, the measured component partial pressure is linearly related to the measured temperature in the flow field, i.e., the measured component partial pressure increases with temperature. Under this condition, the relationship between the temperature and the component partial pressure may be consistent with the relationship represented by a certain contour line in the line damping parameter distribution diagram in Figure $2 b$, i.e., the line damping parameter is the same in the flow field but the normalized coefficient changes. At $n=2$, the line damping parameters of the two quasi-uniform flow fields are equal, and the normalized coefficient ratio $u=x_{1}^{\prime}{ }_{1} / x_{2}^{\prime}$ changes.

Figure 5 shows the absolute value distribution of the error when the line damping parameters of the two quasi-uniform flow fields are equal and set at $0.1 \sim 10$, the normalized coefficient ratio is set at $0.25 \sim 4$, and the IA ratio $A_{1} / A_{2}$ is set at 1:0.2, 1:0.4, 1:0.6, and 1:0.8.

In general, the normalization coefficient ratios resulting from temperature changes are in the range of 0.5-2.0. Figure 5 shows that the IA error generated by the nonuniformity is basically within 0.01 and has a maximum value of about 0.05 . This result shows that TDLAS technology has good applicability in such nonuniform flow fields. 
(a) $\mathrm{A}_{1}: \mathrm{A}_{2}=1: 0.2$
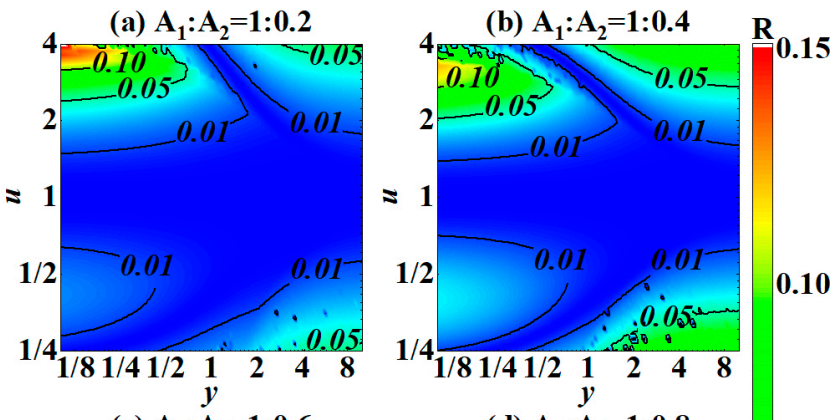

(c) $\mathrm{A}_{1}: \mathrm{A}_{2}=1: 0.6$
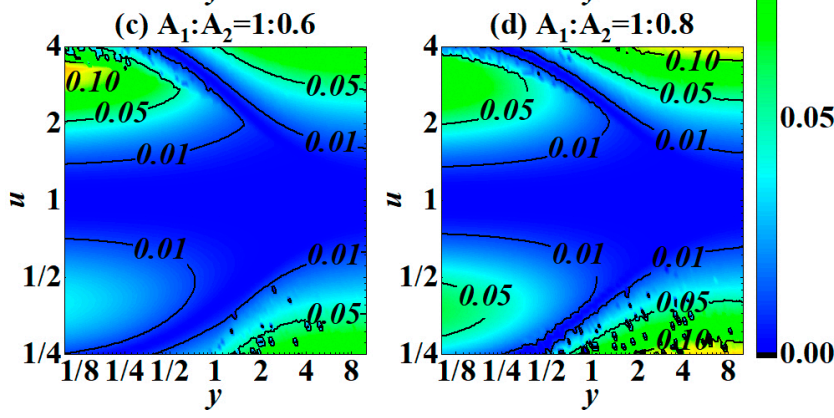

Figure 5. Distribution of the absolute value of the IA error for $y_{1}=y_{2}, u=x_{1}^{\prime}{ }_{1} / x^{\prime}{ }_{2}$, and different values of $A_{1} / A_{2}:$ (a) $A_{1} / A_{2}=1: 0.2$, (b) $A_{1} / A_{2}=1: 0.4$, (c) $A_{1} / A_{2}=1: 0.6$, (d) $A_{1} / A_{2}=1: 0.8$.

\subsection{Effect of the Line-Shape Function}

The analysis presented in Section 2 revealed that the line-shape function directly affects the fitting result. For the Voigt line shape, in addition to the mentioned 916 algorithm and Fadf algorithm approximations, the linear approximation method proposed by Whiting is widely used [37]. In the situation that the line damping parameter is too large or too small, researchers usually choose a Gaussian or Lorentz line shape to fit the absorption signal. Although there are many attempts of using different fitting algorithms used, the evaluation of fitting algorithms is beyond the scope of this work. We believe that the four methods above would well represent the influence of the fitting algorithm on the error. Figure 6 shows the calculated error distribution when using these four line-shape fitting functions for $n=2, A_{1} / A_{2}=1,0.25 \leqslant u \leqslant 4$, and $0.1 \leqslant y \leqslant 10$.
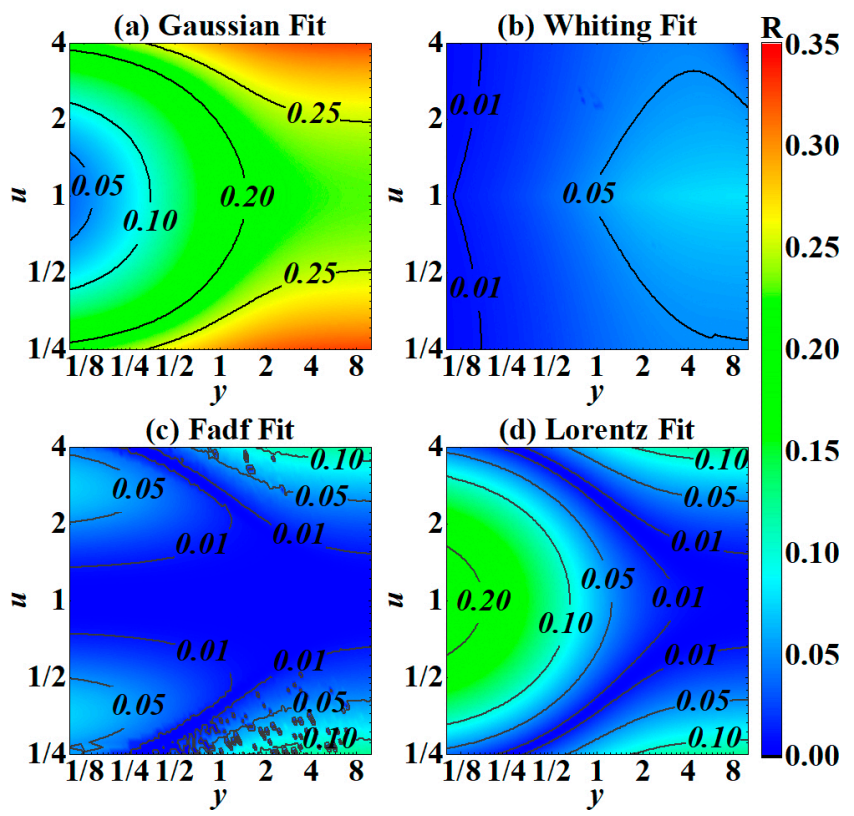

Figure 6. Distribution of the absolute value of the IA error calculated using the four fitting functions: (a) Gaussian Fit, (b) Whiting Fit, (c) Fadf Fit, (d) Lorentz Fit. 
The error distributions in the four cloud images are basically symmetrical across the $u=1$ axis. This symmetry arises because the two normalized coefficient ratios $u$ and $1 / u$ are analytically equivalent when the IA ratio is 1 . The calculation result supports the conclusion to the analysis in Section 2.2.3.

The Fadf algorithm can be used as a representative of the Voigt line-shape highprecision approximation algorithm. The error is small $(R<0.01)$ when the flow field is relatively uniform $(u \rightarrow 1)$ and large $(R>0.05)$ when the flow field is very uneven ( $u>2$ or $u<0.5$ ). Most of the errors obtained when using the Whiting approximation function, as a linear approximation method, are greater than 0.01 , even in a uniform situation, and the error can exceed 0.05. The error obtained when using the numerical approximation algorithm is closer to that for the Voigt theoretical model than that for the linear approximation method.

A comparison of the Gaussian, Lorentz, and Fadf fitting results reveals that in the area $y<0.25$, the error generated by the Gaussian fitting is closer to but larger than the error generated by the Voigt fitting. In the area in which $y>1$ and $R>0.01$, the error generated by the Lorentz fitting is basically the same as that generated by the Voigt fitting, and there is no black noise on the Lorentz fitting error distribution graph. It is reasonably inferred that in this area, the fitting results obtained using the Lorentz function and the Voigt function should be consistent. The black noise point for the Fadf fit is due to the numerical approximation of the Voigt line-shape by the algorithm. So, the black noise point data for the Fadf fit can be replaced by the fitting result of the Lorentz function.

\section{Discussion and Application}

\subsection{Effect of the Absorption Line Overlap}

As discussed in Section 2, the above analysis is based on isolated absorption lines. In most practical applications, there may also be an absorption line overlap. The target absorption line overlaps with the weaker surrounding absorption line, distorting the absorption signal. This problem arises regardless of whether the flow field is uniform. To analyze the coupling effect of nonuniformity and absorption line overlap, this section takes two typical applications (i.e., the swirling flame and inlet oblique shock wave $[10,11]$ ) as examples and uses the method described in Section 2.

Figure 7 is a schematic diagram of the working conditions. Both examples can be simplified as a combination of two quasi-uniform flow field areas. In the swirling flame, the red circle divides the high-temperature zone from the low-temperature zone, the static pressure is constant, and the component concentration is linearly related to the temperature. In the inlet oblique shock wave, the shock wave divides the high-temperature zone from the low-temperature zone. The component concentration is constant, and the pressure changes drastically. $L$ is the total absorption optical path length of the laser in the flow field, $\delta$ and $c$ are the absorption optical path lengths in the hot zone and the cold zone, respectively. The Mach number (Ma) is equal to 2.5 , and the component concentration $(\mathrm{X})$ is equal to 0.1 , in the case of the inlet oblique shock wave. The physical parameters of the two flow fields are given in Table 1.
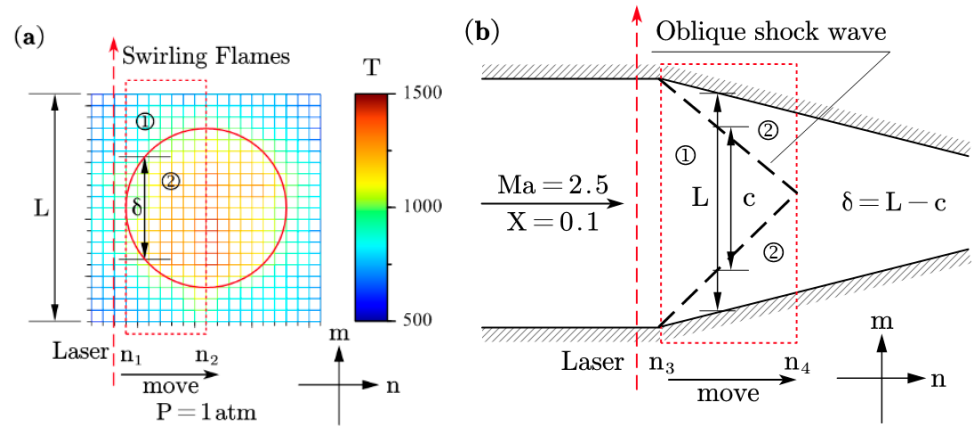

Figure 7. Schematic of the case working conditions: (a) swirl flame and (b) inlet oblique shock. 
Table 1. Physical parameters of the flow field under two working conditions.

\begin{tabular}{ccccc}
\hline Work Condition & Flow Field & $\begin{array}{c}\text { Temperature } \\
\text { (K) }\end{array}$ & \multicolumn{2}{c}{ Water Partial Pressure (Atm) } \\
\hline & & & $\begin{array}{c}\text { Static Pressure } \\
\text { (Atm) }\end{array}$ & Concentration \\
\hline Swirling Flames & 11 & 500 & 1.0 & 0.015 \\
Oblique shock wave & 22 & 1500 & 1.0 & 0.045 \\
& 11 & 900 & 1.0 & 0.1 \\
& 22 & 1440 & 4.0 & 0.1 \\
\hline
\end{tabular}

Four widely used absorption lines of water vapor $[10,43,44]$ at $6807.8,7185.6,7444.3$, and $7466.3 \mathrm{~cm}^{-1}$ are analyzed. According to the HITRAN database [29], there are more than a dozen absorption lines of water within $1 \mathrm{~cm}^{-1}$ around the four target lines. These adjacent absorption lines interfere with the target lines to varying degrees. They are thus used to illustrate the effect of line-shape distortion generated by absorption line overlap on the IA.

Suppose that there is a laser beam that includes the four wavenumber ranges shown in Figure 8. As shown in Figure 7, the laser moves $n_{1} \rightarrow n_{2}$ in the case of the swirling flame and $n_{3} \rightarrow n_{4}$ in the case of the inlet oblique shock wave. As $\delta / L$ (which is defined as the ratio of the length of the high temperature zone to the total absorption optical path) changes, the IA errors in the isolated (target absorption line) and blended (all absorption lines within the wave number range) spectra are calculated.

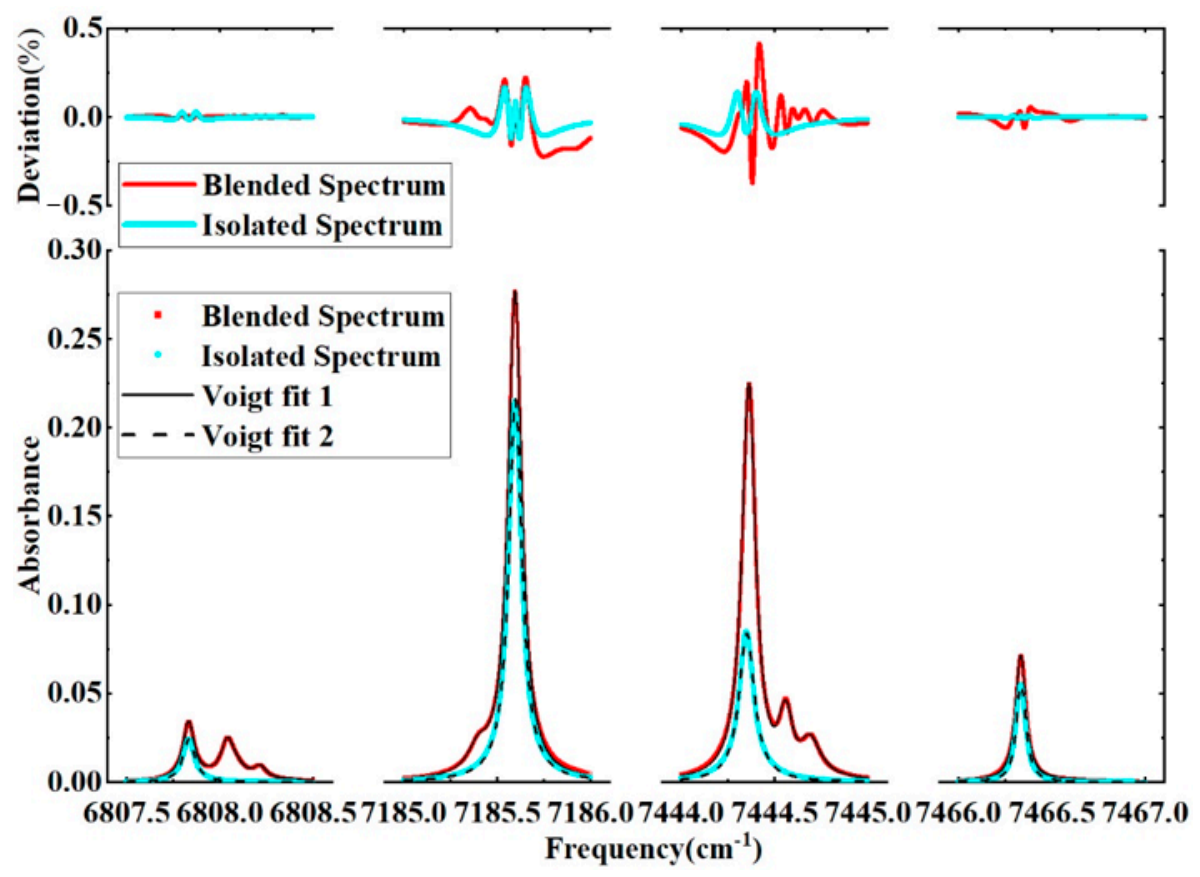

Figure 8. Schematic diagram of the absorption signal simulation and fitting.

Figure 8 is a schematic diagram of the absorption signal simulation and fitting when the laser passes through any of the flow fields in Figure 7. The red line at the bottom is the simulated blended spectrum, and the black solid line is the result of the multi-peak fitting of the simulated blended spectrum. The blue line is the simulated isolated spectrum, and the black dashed line is the result of the single-peak fitting of the simulated isolated spectrum. Here, the isolated spectrum is assumed to exist. This is to compare with the blended spectrum to analyze the effect of the absorption line overlap. The red and blue lines at the top are the fitting residuals of the two absorption signals. The difference between the two residuals shows the effect of the absorption line overlap on the line-shape fitting. 
Figure 8 shows that the multiple absorption lines in the four wavenumber ranges overlap to form a blended spectrum. The right wing of the absorption line at $6807.8 \mathrm{~cm}^{-1}$ has two peaks that formed through the overlapping of several absorption lines. There is a small absorption peak that formed through the overlapping of several absorption lines on the left wing of the absorption line at $7185.3 \mathrm{~cm}^{-1}$. The absorption peak at $7444.3 \mathrm{~cm}^{-1}$ formed through the overlapping of three approximate strength absorption lines, and there are two absorption peaks on its right wing. The absorption peak at $7466.3 \mathrm{~cm}^{-1}$ formed through the overlapping of four absorption lines. Among the four lines, the line at $7185.3 \mathrm{~cm}^{-1}$ has the best "isolation" and is followed by the line at $6807.8 \mathrm{~cm}^{-1}$. The isolation of the absorption peak at $7444.3 \mathrm{~cm}^{-1}$ is poor and that of the absorption peak at $7466.3 \mathrm{~cm}^{-1}$ is the worst.

Figure $9 \mathrm{a}, \mathrm{b}$ presents the calculation results of IA error under two conditions. The red and black lines show IA errors of isolated and blended peaks, respectively. Overall, the trends of the black and red lines are the same for the same absorption line and the same working condition. The isolated peak error better characterizes the IA error when the isolation of the absorption line is better, and the error generated by the nonuniformity is larger. The IA error is more affected by the absorption line overlap when the isolation of the absorption line is worse or the error for the isolated peak is smaller.

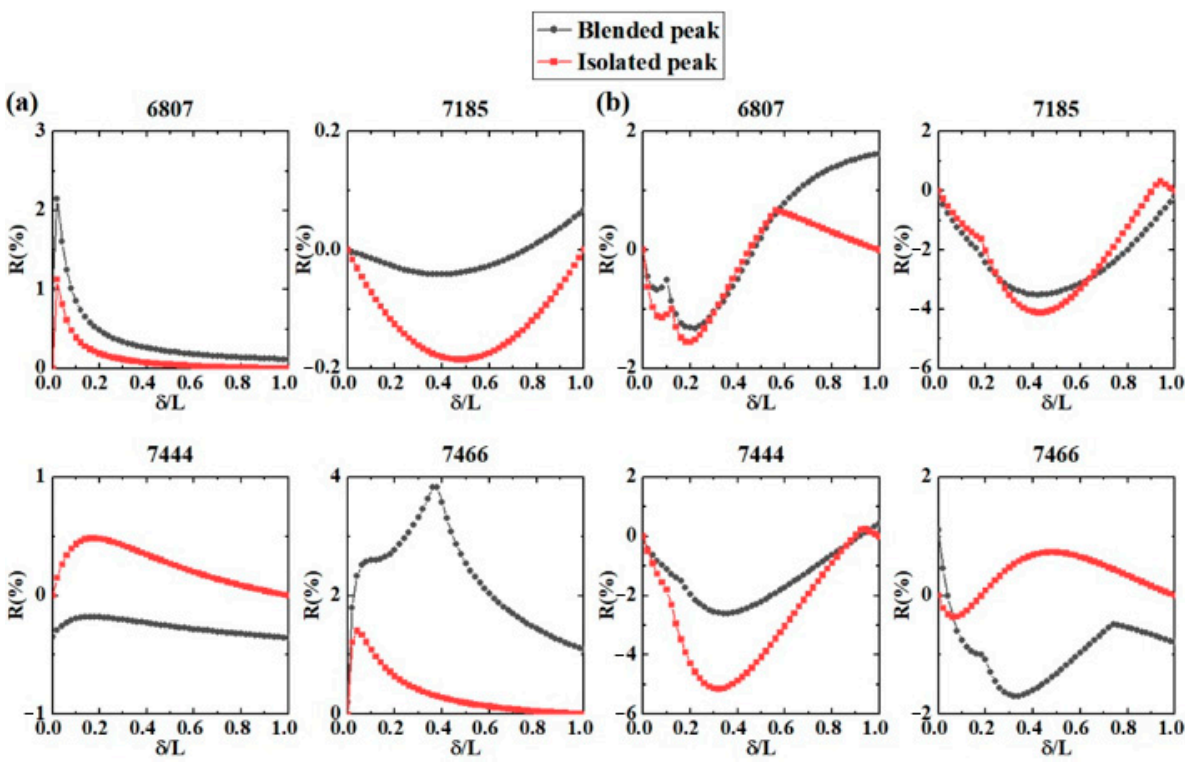

Figure 9. Four absorption line error distribution results. The IA error changes as $\delta / L$ (which is defined as the proportion of high temperature zone) changes. (a) swirl flame and (b) inlet oblique shock.

In the case of the shock wave, the oblique shock wave generates a pressure and velocity gradient along the optical path, resulting in a frequency shift $v_{c}$ at the center of the peak. According to a calculation based on spectral parameters, the frequency shift generated by the pressure and velocity is $v_{c}<0.001 \mathrm{~cm}^{-1}$, which can be ignored in the analysis. The effect of the frequency shift can be added following this work if needed.

\subsection{Method of Estimating Nonuniformity Error}

The method of estimating the IA error for a nonuniform flow field is established on the basis of the analysis and discussion above. We summarize the steps as follows:

(1). Firstly, the nonuniform flow field is simplified as a combination of a few quasi-uniform flow field regions.

(2). Secondly, in each region of the quasi-uniform flow field, the line damping parameters, normalization coefficient, and IA are calculated according to the physical and spectral parameters of the flow field. 
(3). Thirdly, find the corresponding error in the error distribution according to the lineshape parameter. If the fitting function used in the measurement is that of the Voigt numerical approximation algorithm, then the error distribution is found in the Supplementary Materials when $n=2$. When $n>2$, the error distribution can be calculated through the analysis described in Section 2.

(4). Finally, the effect of isolation on the errors needs to be considered. When the isolation of an absorption line is poor, it is necessary to calculate the IA error generated by the absorption line overlap under a uniform flow field. The two errors are added together to obtain the estimated IA error under the nonuniform flow field.

It is noted that considering the quasi-uniform flow field division and the isolation of the absorption line, the influence of the uncertainty of other parameters (including pressure, temperature, concentration, and line parameters) can be ignored. The estimated error is larger than the actual error.

\section{Conclusions}

This work studied the IA error of TDLAS technology in the case of a nonuniform flow field. The IA error was quantified using line-shape parameters (i.e., the line damping parameter $y_{i}$, normalization coefficient $x^{\prime}{ }_{i}$, and IA $A_{i}$ ). See Supplementary Materials for the IA error distribution table. A general method of estimating the IA error for the nonuniform flow field was given. The three main findings of the present study are summarized as follows.

First, the error is affected by the coupling of the flow field nonuniformity, and the absorption line features. For any isolated absorption line, the error is basically less than 0.01 when the physical environment of the flow field does not change drastically. The error may exceed 0.1 when the temperature or pressure gradient is large. An absorption line overlap affects the magnitude of the IA error for poorly isolated absorption lines. However, the absorption line overlap hardly affects the overall error distribution.

Second, the IA error is basically less than 0.05 for a flow field in which the temperature and the measured partial pressure have a specific linear relationship. TDLAS technology has good applicability in such nonuniform flow fields.

Third, the fitting function has an important effect on the IA error. The IA error obtained using the numerical approximation algorithm is closer to that of the Voigt theoretical model than that of the linear approximation method. When all line damping parameters of the nonuniform flow field are greater than 1 , the effect of using the Lorentz function for fitting is basically the same as that of using the numerical approximation of the Voigt function for fitting.

This work illustrated the applicability of TDLAS technology in nonuniform flow fields and provided input parameters for the evaluation of TDLAT measurement error.

Supplementary Materials: The following are available online at https:/ /www.mdpi.com/article/10 .3390/app112210936/s1, Integrated Absorbance Error Distribution Table for the IA error distribution table.

Author Contributions: Conceptualization, F.L.; software, R.L.; validation, X.L.; data curation, R.L.; writing—original draft preparation, R.L.; writing—review and editing, X.L.; project administration, X.Y. All authors have read and agreed to the published version of the manuscript.

Funding: This research was funded by National Natural Science Foundation of China, grant number 12072355, 11802315, 11927803 and Youth Innovation Promotion Association of CAS, grant number 2018023.

Institutional Review Board Statement: Not applicable.

Informed Consent Statement: Not applicable.

Data Availability Statement: Data supporting the results of this work can be obtained from the corresponding author upon reasonable request. 
Acknowledgments: This work was funded by National Natural Science Foundation of China, grant number 12072355, 11802315, 11927803 and Youth Innovation Promotion Association of CAS, grant number 2018023.

Conflicts of Interest: The authors declare no conflict of interest.

\section{References}

1. Hanson, R.K. Applications of quantitative laser sensors to kinetics, propulsion and practical energy systems. Proc. Combust. Inst. 2011, 33, 1-40. [CrossRef]

2. Goldenstein, C.S.; Spearrin, R.M.; Jeffries, J.B.; Hanson, R.K. Infrared laser-absorption sensing for combustion gases. Prog. Energy Combust. Sci. 2017, 60, 132-176. [CrossRef]

3. Liu, C.; Xu, L. Laser absorption spectroscopy for combustion diagnosis in reactive flows: A review. Appl. Spectrosc. Rev. 2019, 54, 1-44. [CrossRef]

4. Rieker, G.; Jeffries, J.; Hanson, R.; Mathur, T.; Gruber, M.; Carter, C. Diode laser-based detection of combustor instabilities with application to a scramjet engine. Proc. Combust. Inst. 2009, 32, 831-838. [CrossRef]

5. $\quad$ Chang, L.S.; Strand, C.L.; Jeffries, J.B.; Hanson, R.K.; Diskin, G.S.; Gaffney, R.L.; Capriotti, D.P. Supersonic Mass-Flux Measurements via Tunable Diode Laser Absorption and Nonuniform Flow Modeling. AIAA J. 2011, 49, 2783-2791. [CrossRef]

6. Sanders, S.T.; Wang, J.; Jeffries, J.B.; Hanson, R.K. Diode-laser absorption sensor for line-of-sight gas temperature distributions. Appl. Opt. 2001, 40, 4404-4415. [CrossRef] [PubMed]

7. Liu, X.; Jeffries, J.B.; Hanson, R.K. Measurement of Non-Uniform Temperature Distributions Using Line-of-Sight Absorption Spectroscopy. AIAA J. 2007, 45, 411-419. [CrossRef]

8. Goldenstein, C.; Schultz, I.A.; Jeffries, J.B.; Hanson, R.K. Two-color absorption spectroscopy strategy for measuring the column density and path average temperature of the absorbing species in nonuniform gases. Appl. Opt. 2013, 52, 7950-7962. [CrossRef] [PubMed]

9. Ma, L.; Li, X.; Sanders, S.T.; Caswell, A.W.; Roy, S.; Plemmons, D.H.; Gord, J.R. 50-kHz-rate 2D imaging of temperature and H ${ }_{2} \mathrm{O}$ concentration at the exhaust plane of a J85 engine using hyperspectral tomography. Opt. Express 2013, 21, 1152-1162. [CrossRef] [PubMed]

10. Li, F.; Kang, G.; Lin, X.; Wang, K.; Yu, X. Diode Laser Absorption Tomography for swirl flames Application. In Fifth International Symposium on Laser Interaction with Matter; Zhao, Y., Ed.; Spie-Int Soc Optical Engineering: Bellingham, WA, USA, 2019.

11. Li, F.; Yu, X.; Gu, H.; Li, Z.; Zhao, Y.; Ma, L.; Chen, L.; Chang, X. Simultaneous measurements of multiple flow parameters for scramjet characterization using tunable diode-laser sensors. Appl. Opt. 2011, 50, 6697-6707. [CrossRef] [PubMed]

12. Wei, C.; Pineda, D.I.; Goldenstein, C.S.; Spearrin, R.M. Tomographic laser absorption imaging of combustion species and temperature in the mid-wave infrared. Opt. Express 2018, 26, 20944-20951. [CrossRef]

13. Liu, C.; $\mathrm{Xu}, \mathrm{L}$;; Cao, Z. Measurement of nonuniform temperature and concentration distributions by combining line-of-sight tunable diode laser absorption spectroscopy with regularization methods. Appl. Opt. 2013, 52, 4827-4842. [CrossRef]

14. Cai, W.; Kaminski, C.F. Tomographic absorption spectroscopy for the study of gas dynamics and reactive flows. Prog. Energy Combust. Sci. 2017, 59, 1-31. [CrossRef]

15. Guha, A.; Schoegl, I. Tomographic laser absorption spectroscopy using Tikhonov regularization. Appl. Opt. 2014, 53, 8095-8103. [CrossRef] [PubMed]

16. Liu, C.; Xu, L.; Chen, J.; Cao, Z.; Lin, Y.; Cai, W. Development of a fan-beam TDLAS-based tomographic sensor for rapid imaging of temperature and gas concentration. Opt. Express 2015, 23, 22494-22511. [CrossRef]

17. Ouyang, X.; Varghese, P.L. Line-of-sight absorption measurements of high temperature gases with thermal and concentration boundary layers. Appl. Opt. 1989, 28, 3979-3984. [CrossRef] [PubMed]

18. Li, F.; Yu, X.; Cai, W.; Ma, L. Uncertainty in velocity measurement based on diode-laser absorption in nonuniform flows. Appl. Opt. 2012, 51, 4788-4797. [CrossRef]

19. Qu, Z.; Werhahn, O.; Ebert, V. Thermal Boundary Layer Effects on Line-of-Sight Tunable Diode Laser Absorption Spectroscopy (TDLAS) Gas Concentration Measurements. Appl. Spectrosc. 2018, 72, 853-862. [CrossRef] [PubMed]

20. Ma, L.; Cheong, K.-P.; Yang, M.; Yuan, C.; Ren, W. On the Quantification of Boundary Layer Effects on Flame Temperature Measurements Using Line-of-sight Absorption Spectroscopy. Combust. Sci. Technol. 2021, 1-18. [CrossRef]

21. Nei, W.; Ye, Q.-H.; Xu, Z.-Y.; Zhang, G.-L.; Xia, H.-H.; Kan, R.-F. Study on the method of voigt profiles two wings fitting non-uniform flow field absorbance. Spectrosc. Spectr. Anal. 2017, 37, 816-821.

22. Pogány, A.; Klein, A.; Ebert, V. Measurement of water vapor line strengths in the 1.4-2.7 mu m range by tunable diode laser absorption spectroscopy. J. Quant. Spectrosc. Radiat. Transf. 2015, 165, 108-122. [CrossRef]

23. Emmert, J.; Blume, N.G.; Dreizler, A.; Wagner, S. Data analysis and uncertainty estimation in supercontinuum laser absorption spectroscopy. Sci Rep. 2018, 8, 10312. [CrossRef]

24. Li, J.; Du, Y.; Peng, Z.; Ding, Y. Measurements of spectroscopic parameters of CO2 transitions for Voigt, Rautian, galatry and speed-dependent voigt profiles near 1.43 mu m using the WM-DAS method. J. Quant. Spectrosc. Radiat. Transf. 2019, 224, 197-205. [CrossRef]

25. Philippe, L.C.; Hanson, R.K. Laser diode wavelength-modulation spectroscopy for simultaneous measurement of temperature, pressure, and velocity in shock-heated oxygen flows. Appl. Opt. 1993, 32, 6090-6103. [CrossRef] [PubMed] 
26. Arroyo, M.P.; Langlois, S.; Hanson, R.K. Diode-laser absorption technique for simultaneous measurements of multiple gasdynamic parameters in high-speed flows containing water vapor. Appl. Opt. 1994, 33, 3296-3307. [CrossRef]

27. Roston, G.; Obaid, F. Exact analytical formula for Voigt spectral line profile. J. Quant. Spectrosc. Radiat. Transf. 2005, 94, 255-263. [CrossRef]

28. Armstrong, B. Spectrum line profiles: The Voigt function. J. Quant. Spectrosc. Radiat. Transf. 1967, 7, 61-88. [CrossRef]

29. Gordon, I.E.; Rothman, L.S.; Hill, C.; Kochanov, R.V.; Tan, Y.; Bernath, P.F.; Birk, M.; Boudon, V.; Campargue, A.; Chance, K.V.; et al. The HITRAN2016 molecular spectroscopic database. J. Quant. Spectrosc. Radiat. Transf. 2017, 203, 3-69. [CrossRef]

30. Zhou, X.; Jeffries, J.; Hanson, R. Development of a fast temperature sensor for combustion gases using a single tunable diode laser. Appl. Phys. 2005, 81, 711-722. [CrossRef]

31. Bendana, F.A.; Sanders, I.C.; Castillo, J.J.; Hagström, C.G.; Pineda, D.I.; Spearrin, R.M. In-situ thermochemical analysis of hybrid rocket fuel oxidation via laser absorption tomography of $\mathrm{CO}, \mathrm{CO}_{2}$, and $\mathrm{H}_{2} \mathrm{O}$. Exp. Fluids 2020,61, 13. [CrossRef]

32. Skrotzki, J.; Habig, J.C.; Ebert, V. Integrative fitting of absorption line profiles with high accuracy, robustness, and speed. Appl. Phys. 2014, 116, 393-406. [CrossRef]

33. Lourakis, M.I. A brief description of the Levenberg-Marquardt algorithm implemented by levmar. In Technical Report; Institute of Computer Science, Foundation for Research and Technology: Hellas/Crete, Greece, 2005.

34. Yin, Z.Q.; Wu, C.; Gong, W.Y.; Gong, Z.K.; Wang, Y.J. Voigt profile function and its maximum. Acta Phys. Sin. 2013, 62, 123301.

35. Kuntz, M. A new implementation of the Humlicek algorithm for the calculation of the Voigt profile function. J. Quant. Spectrosc. Radiat. Transf. 1997, 57, 819-824. [CrossRef]

36. Letchworth, K.L.; Benner, D.C. Rapid and accurate calculation of the Voigt function. J. Quant. Spectrosc. Radiat. Transf. 2007, 107, 173-192. [CrossRef]

37. Whiting, E. An empirical approximation to the Voigt profile. J. Quant. Spectrosc. Radiat. Transf. 1968, 8, 1379-1384. [CrossRef]

38. Zaghloul, M.R.; Ali, A.N. Algorithm 916: Computing the Faddeyeva and Voigt Functions. ACM Trans. Math. Softw. 2011, 38, 22. [CrossRef]

39. Gautschi, W. Efficient Computation of the Complex Error Function. SIAM J. Numer. Anal. 1970, 7, 187-198. [CrossRef]

40. Abrarov, S.M.; Quine, B.M. On the Fourier expansion method for highly accurate computation of the Voigt/complex error function in a rapid algorithm. arXiv 2012, arXiv:1205.1768.

41. Abrarov, S.M.; Quine, B.M. Efficient algorithmic implementation of the Voigt/complex error function based on exponential series approximation. Appl. Math. Comput. 2011, 218, 1894-1902. [CrossRef]

42. Abrarov, S.M.; Quine, B.M. A rational approximation for efficient computation of the Voigt function in quantitative spectroscopy. arXiv 2015, arXiv:1504.00322. [CrossRef]

43. Thomas, J.J.; Eveland, W.C.; Medzon, E.L.; Christian, W.; Wylie, D.E.; Burckhalter, J.H.; Jones, R.H. Hydrolysis of Phthalyl Amino Acid Esters of Fluoroscein in the Presence of Leucine Aminopeptidase. Exp. Biol. Med. 1972, 140, 179-182. [CrossRef] [PubMed]

44. Liu, C.; Xu, L.; Cao, Z.; McCann, H. Reconstruction of Axisymmetric Temperature and Gas Concentration Distributions by Combining Fan-Beam TDLAS With Onion-Peeling Deconvolution. IEEE Trans. Instrum. Meas. 2014, 63, 3067-3075. [CrossRef] 